International Journal of Ophthalmology \& Eye Science (IJOES)

ISSN 2332-290X

\title{
Aspirin and its Metabolites Enhance the Expression of Vascular Endothelial Growth Factor in Retinal Pigment Epithelial Cell Cultures - Implications in the Pathophysiology of Age-Related Macular Degeneration
}

\author{
Iqbal $\mathrm{O}^{1 *}$, Brambl W'${ }^{2}$, Ottman A $\mathrm{O}^{2}$,Malley $\mathrm{P}^{6}$, Gaynes $\mathrm{J}^{4}$, De Alba F${ }^{3}$, Gaynes $\mathrm{B}^{3}$, Fareed J ${ }^{5}$, Bouchard CS \\ ${ }^{1}$ Professor of Pathology \& Ophthalmology, Loyola University Medical Center, USA. \\ ${ }^{2}$ Research Scholar, Stritch School of Medicine, Loyola University Chicago, USA. \\ ${ }^{3}$ Professor of Pathology \& Ophthalmology, Loyola University Medical Center, USA. \\ ${ }^{4}$ Research Assistant, Masonic Cancer Center, University of Minnesota, USA. \\ Professor, Department of Pharmacology \& Pathology, Loyola University Health System, Maywood, USA. \\ ${ }^{6}$ Research Scholar, Department of Ophthalmology, Loyola University Medical Center, USA. \\ Professor of Ophthalmology, Loyola University Health System, Maywood, USA.
}

\begin{abstract}
Purpose: An estimated 19.3\% of adults, especially the elderly in the United States regularly use Aspirin for cardioprotection. Recently, multiple cohort studies have concluded that regular aspirin use for 10-15 years was associated with a statistically significant increase in the risk of incident age-related and neovascular acute macular degeneration. It has been hypothesized that aspirin or its metabolites induce the expression of vascular endothelial growth factor (VEGF).

Materials \& Methods: Retinal pigment epithelial cells, ARPE-19 (ATCC ${ }^{\mathbb{C}}$ CRL-2302 ${ }^{\mathrm{TM}}$ ) were cultured. The cells were grown to achieve $95 \%$ confluence and then the media was changed. Cells cultured under blue light, red light, or darkness were subjected to a challenge with high dose aspirin $(0.925 \mathrm{mg} / \mathrm{dL})$, low dose aspirin $(0.325 \mathrm{mg} / \mathrm{dL})$, or hippuric acid $(0.325$ $\mathrm{mg} / \mathrm{dL}$ ). Light was generated using 2 red or blue LEDs powered by 3v CR2032 batteries. The 24 -well plate was incubated with or without drugs in blue light, red light or darkness at 37C for 16 hours. The supernatants were harvested, and VEGF was quantified. One-way ANOVA using Dunnett's multiple comparison test was performed to analyze statistical significance.

Results: Cells exposed to blue light or darkness and hippuric acid showed a statistically significant increase in VEGF secretion $(\mathrm{P}=0.0012)$. However, cells exposed to red light with hippuric acid challenge showed no significant difference from the mean of cells exposed to darkness and sham control.

Conclusions: Retinal pigment epithelial cells challenged with oxidative stress provided by blue light or darkness in the presence of hippuric acid increased VEGF secretion, suggesting a possible cause for neovascularization in age-related macular degeneration. RPE cells exposed to red light, known to abrogate oxidative stress, had decreased levels of VEGF induction by hippuric acid.
\end{abstract}

\section{*Corresponding Author:}

Omer Iqbal MD,

Professor Department of Pathology \& Ophthalmology, $2160 \mathrm{~S} 1^{\text {st }} \mathrm{Av}-$ enue Building 102 Room 2646 Maywood Il 60153. USA.

Tel: (708) 216-5587

E-mail: oiqbal@luc.edu

Received: June 23, 2015

Accepted: August 22, 2015

Published: August 25, 2015

Citation: Iqbal O, et al., (2015) Aspirin and its Metabolites Enhance the Expression of Vascular Endothelial Growth Factor in Retinal Pigment Epithelial Cell Cultures - Implications in the Pathophysiology of AgeRelated Macular Degeneration. Int J Opbthalmol Eye Res 03(7), 115-120. doi: http://dx.doi.org/10.19070/2332-290X-1500025

Copyright: Iqbal $\mathbf{O}^{\circ}$ 2015. This is an open-access article distributed under the terms of the Creative Commons Attribution License, which permits unrestricted use, distribution and reproduction in any medium, provided the original author and source are credited.

\section{Introduction}

Age-related macular degeneration (AMD) is the leading cause of adult eye disease among industrialized nations, particularly in the elderly [1]. Clinically, there is a critical unmet need in the treatment of this condition essentially due to the lack of proper understanding of disease pathophysiology. The US, Canada, and Cuba spend approximately US\$98 Billion in direct healthcare costs for visual impairment due to AMD. The global estimate on AMD healthcare spending is US\$255 billion [2]. AMD is a degenerative disease of the central portion of the retina, known as the macula, which results in loss of central vision. Because central vision is required for a majority of daily activities, it significantly impacts the functional status and quality of life. In general, depending on the progression of the disease, AMD is either characterized as dry type or wet type. The pathogenesis is currently poorly understood; however, ischemia and oxidative stress are believed to be the key factors involved. This hypoxia state results in the release of factors such as Vascular Endothelial Growth Factor (VEGF), and inflammatory signals, which help the growth of new and ab- 
normal vessels [3]. (Figure 1) This is especially true when attempting to explain the pathogenesis of wet type AMD, also known as choroidal neovascularization, because clinical observations show that there is growth of new and abnormal vessels into the subretinal space [4]. In particular, VEGF release due to retinal ischemia results in weaker vessels that grow behind the retina and under the macula. As a result of these weaker walls, these vessels begin to leak blood and fluid, causing the macula to swell and eventually progressing to permanent damage of central vision of the eye. Several other etiologies have been discussed as well, such as the complement pathway, single nucleotide polymorphisms, macrophages, etc [3]. In addition, there are also several risk factors for AMD, including, age $(>50)[5,6]$, smoking [7], family history [8], and cardiovascular disease [9]. AMD is considered to be a multifactorial disease in which several factors including oxidative damage, inflammatory reaction and abnormal immune responses may be involved [10-12]. The retinal stress may in part result from exposure of the eye to visible blue light. The combination of blue light exposure and low plasma concentrations of antioxidants was found to be associated with the early stages of AMD; furthermore, blue light exposure in middle age individuals might be more damaging than at younger ages, as per the results of a recent European Eye Study (EUROEYE) [13]. Recent reports indicate that our eyes are increasingly being exposed to light from light emitting diodes (LED's) and light of video display terminals (VDT's) which contain blue light [14]. The putative pathway of blue LED light-induced retinal photoreceptor-derived cell damage is shown in Figure 1. Although blue light causes oxidative stress, red light is known to reduce oxidative stress [15].

Several studies have shown the association of aspirin use with age-related macular degeneration [16]. Recently, a 15-year prospective analysis of data from an Australian population-based cohort study concluded that regular aspirin use was associated with a 2.5-fold greater risk of incidental neovascular AMD, independent of a history of cardiovascular disease or smoking [17]. An article by Klein, et al found that chronic use of aspirin for at least 10 years increased the risk of neovascular AMD [18]. It comments on how aspirin's mechanism of action on retinal vessels may be different than the mechanism of action responsible for cardioprotection. However, another study investigating the possible relationship of aspirin and age-related macular degeneration concluded that the relationship between aspirin use and AMD could not be established [19]. Aspirin and hippuric acid, the glycine conjugate of benzoic acid, share common metabolites such as 2-3-dihydroxybenzoic acid and 3-hydroxybenzoic acid (Figure 2). Furthermore, we based our experimental concentrations of aspirin on the average circulating level of aspirin in plasma after regular dose of aspirin.

Despite all the research so far, the mechanism of how aspirin causes neovascularization is not clearly understood. Although, it is known that aspirin enhances choroidal neovascularization in the presence of cell injury, it is not clear as to what causes cell injury or how retinal ischemia occurs. We hypothesize that while the cell is under oxidative stress and cell injury, aspirin and its toxic metabolites trigger VEGF expression leading to choroidal neovascularization.

\section{Materials and Methods}

ARPE-19 (ATCC ${ }^{\circledR}$ CRL-2302 ${ }^{\text {TM }}$ ) cells were kindly provided by Dr. Knepper. RPE cells were cultured in DMEM medium, 10\%
FBS, Penicillin, Streptomycin, Ciprofloxacin at $37^{\circ} \mathrm{C}, \% 5 \mathrm{CO}_{2}$. RPE Cells were tyrpsinized and plated in a 24 well plate in a total of $500 \mu \mathrm{L}$ of DMEM at 40,000 cells $/ \mathrm{mL}$. This concentration was used because it allows for sustained proliferation. The cells were grown out to $>95 \%$ confluency in about 72 hours and then media was changed. Cells cultured in Blue light, red light, or darkness, were exposed to either sham, $0.925 \mathrm{mg} / \mathrm{dL}$ of aspirin (high dose), $0.325 \mathrm{mg} / \mathrm{dL}$ aspirin (low dose), $0.325 \mathrm{mg} / \mathrm{dL}$ hippuric acid. Light was generated using 2 red or 2 blue LED's powered by 3v CR2032 batteries attached to the inside of a standard freezer box. In this way it was possible to keep the 24 well plates in darkness, red light, or blue light for the period of the experiment. The plates were incubated with or without drugs in blue light, red light, or no light overnight for 16 hours. The supernatants were harvested and VEGF was quantified using Quantikine ELISA Human VEGF Immunoassay kit (R\&D Systems, Inc.), as per manufactures directions. After the supernatants were collected, cells in each individual well were trypsinized, and live cells were counted manually using a hemocytometer. Live cells were identified by the presence of an intact nucleus and cellular membrane under polarized light. Total supernatant protein concentration was determined using modified Lowry method. During analysis of data, VEGF concentration was normalized to the total protein concentration. One-way ANOVA using Dunnett's multiple comparison test was performed using GraphPad Prism.

\section{Results}

The supernatants of ARPE-19 (ATCC ${ }^{\circledR}$ CRL-2302 ${ }^{\text {TM }}$ cells cultured in Blue light, red light, or darkness, and exposed to either sham, $0.925 \mathrm{mg} / \mathrm{dL}$ of aspirin (high dose), $0.325 \mathrm{mg} / \mathrm{dL}$ aspirin (low dose), $0.325 \mathrm{mg} / \mathrm{dL}$ hippuric acid were analyzed to determine the VEGF in triplicates. The data was statistically analyzed to determine the mean, standard deviations, and p-values.

\section{Under Blue Light}

Under the influence of blue light, the low dose aspirin, high dose aspirin and hippuric acid expressed VEGF amounting to $97.83 \pm$ $5.6,104.2 \pm 7.5$ and $114.7 \pm 3.3 \mathrm{pg} / \mathrm{ml}$ respectively, when compared to the sham control value of $105.79 \pm 8.4 \mathrm{pg} / \mathrm{ml}$. Cells exposed to blue light and hippuric acid had higher levels of VEGF expression compared to the darkness sham control and the low and high dose aspirin under similar conditions. (See Figure 3).

\section{Under Darkness}

Under the influence of darkness, the low dose aspirin, and high dose aspirin expressed VEGF amounting to $98.2 \pm 1.68$, and $100.7 \pm 9.0$ respectively when compared to a sham control value of $116.8 \pm 3.1 \mathrm{pg} / \mathrm{ml}$ and a no light control value of $100.7 \pm 7.3$ $\mathrm{pg} / \mathrm{ml}$. Cells exposed to darkness and hippuric acid had higher levels of VEGF expression compared to the darkness sham control and the low and high dose aspirin under similar conditions (See Figure 3).

\section{Under Red Light}

Under the influence of red light, low dose aspirin, high dose aspirin and hippuric acid expressed VEGF amounting to 101.7 $\pm 3.84,96.0 .3 .8$ and $100.8 \pm 0.41 \mathrm{pg} / \mathrm{ml}$ when compared to the sham control value of $95.2 \pm 5.9 \mathrm{pg} / \mathrm{ml}$. Cells exposed to red light and hippuric acid did not show an increased induction of VEGF 
Figure 1. The putative pathway of blue LED light-induced retinal photoreceptor-derived cell damage [14] (Courtesy: Kuse Y et al - Reproduced with kind permission from Dr. Hideaki Hara)

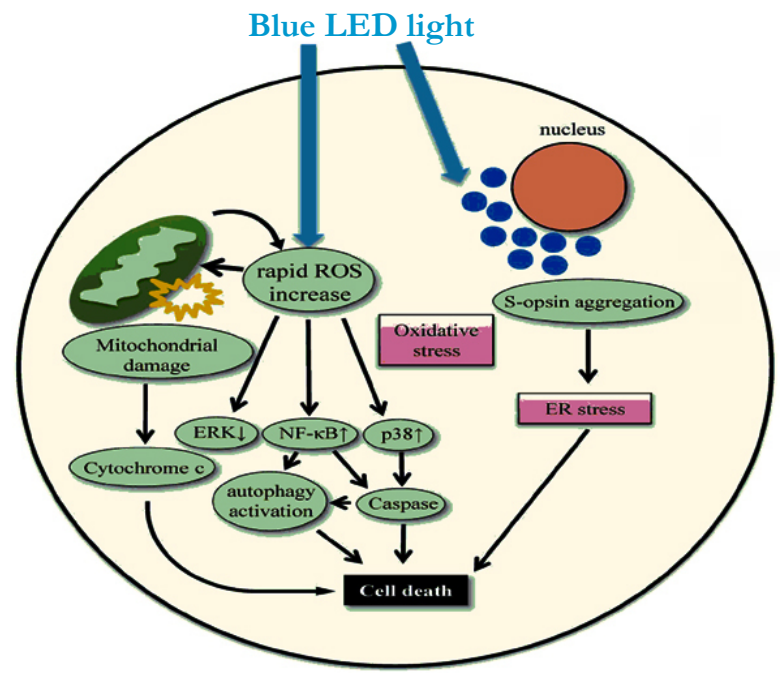

Figure 2: Aspirin and its metabolites.

Links between Aspirin (1) metabolites (black arrows), with two potential metabolites (dashed black arrows) and benzoic acid (2) and hippuric acid (3) metabolites (blue arrows) reported to date in the literature. Metabolites originating from the benzoate degredation via hydroxylation pathway of the human detoxification process are also represented (yellow arrows)

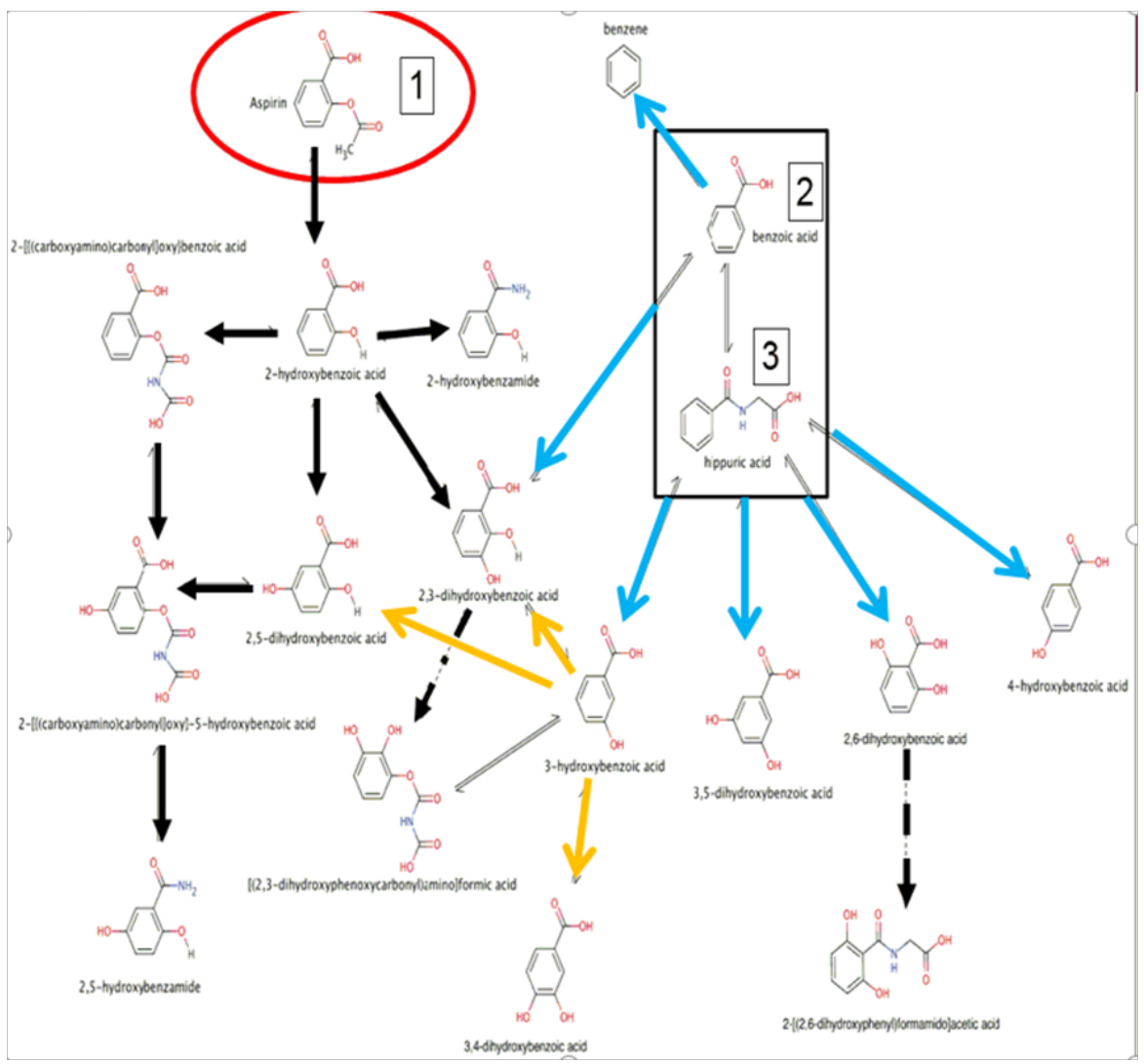

expression when compared to cells exposed to hippuric acid and blue light or darkness (See Figure 3).

\section{Difference in survival of RPE cells}

There was no statistically significant difference in the number of live cells between any experimental group at the conclusion of the experiment as determined by manual hemocytometer counting after they were trypsinized and collected $(\mathrm{p}=0.7274)$. (See supplemental Figure).

\section{Discussion}

We selected Retinal Pigment Epithelial Cells (RPE) for the following reasons. RPE cells play a critical role in the development and maintenance of adjacent photoreceptors. These photoreceptors generate a number of reactive oxygen species when exposed to light, and their proliferation is an important step in the pathogenesis of ocular diseases such as AMD. It is presumed that the metabolites of hippuric acid induce the generation of reactive oxygen species during metabolism as well as induce VEGF expression, leading to choroidal neovascularization. VEGF is a di- 
Figure 3. VEGF Secretion.

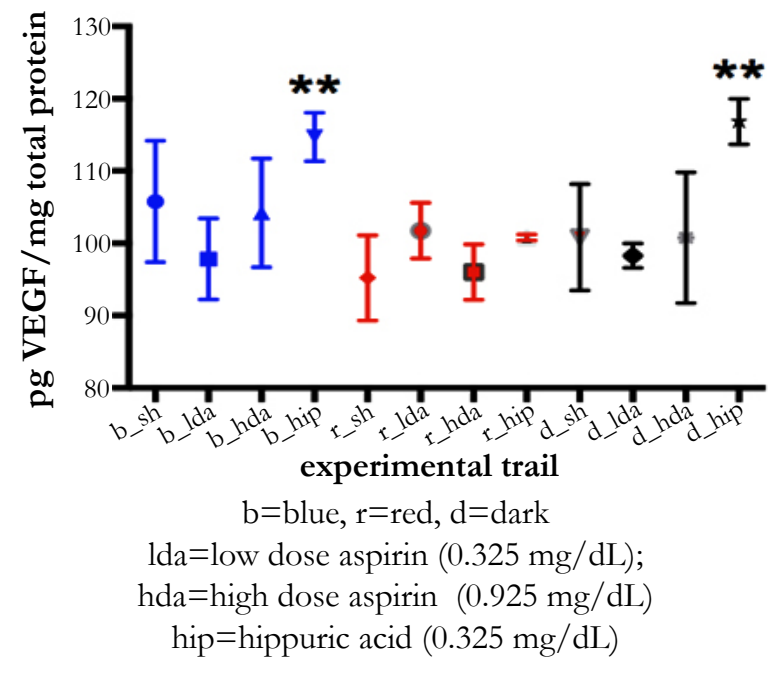

Supplementary Table 1. Raw Data VEGF expression.

\begin{tabular}{|c|c|c|c|c|c|}
\hline (pg VEGF/ mg protein) & Trail 1 & Trail 2 & Trail 3 & Average & Stdev \\
\hline Blue Sham & 115.46613 & 101.667334 & 100.24127 & 105.791578 & 8.40869405 \\
\hline Blue Low Dose Aspirin & 94.7225614 & 94.4619837 & 104.310345 & 97.83163 & 5.61224419 \\
\hline Blue High Aspirin & 103.602129 & 97.005261 & 112.030075 & 104.212488 & 7.53098031 \\
\hline Blue Hippuric Acid & 118.161018 & 114.538441 & 111.457117 & 114.718859 & 3.35559025 \\
\hline Red Sham & 97.7408009 & 88.4605694 & 99.4295635 & 95.2103113 & 5.90611895 \\
\hline Red Low Dose Aspirin & 100.873485 & 98.3948636 & 105.942857 & 101.737069 & 3.84738662 \\
\hline Red High Dose Aspirin & 93.804262 & 100.436508 & 93.7777778 & 96.0061826 & 3.83679715 \\
\hline Red Hippuric Acid & 100.750518 & 101.273378 & 100.452381 & 100.825426 & 0.4155931 \\
\hline Dark Sham & 109.023268 & 94.7538337 & 98.7406533 & 100.839252 & 7.36255922 \\
\hline Dark Low Aspirin & 97.9003705 & 96.8163265 & 100.118718 & 98.2784716 & 1.68335006 \\
\hline Dark High Aspirin & 92.3864102 & 99.537415 & 110.340136 & 100.754654 & 9.0385464 \\
\hline Dark Hippuric Acid & 119.28839 & 117.897727 & 113.314142 & 116.83342 & 3.12609537 \\
\hline
\end{tabular}

Cells exposed to blue light or darkness and hippuric acid showed a statistically significant increase in VEGF secretion ( $\mathrm{P}=0.0012)$ using an ordinary 1 way ANOVA. However, cells exposed to red light with hippuric acid challenge showed no significant difference from the mean of cells exposed to darkness and sham control.

Supplementary Figure. Live cell counts after experiment.

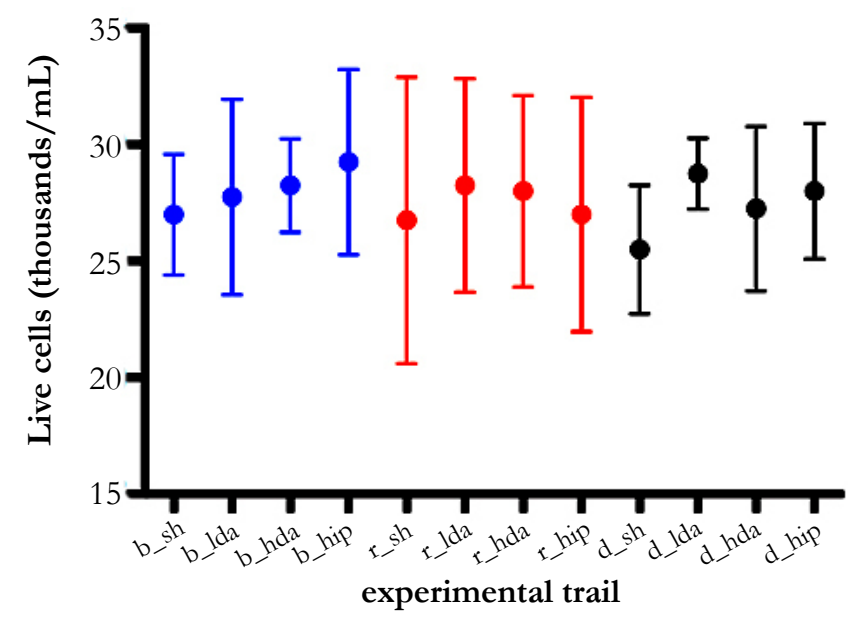

Graphs shown with mean live cell count in thousand $/ \mathrm{mL}$ (circle) and $95 \%$ confidence intervals. Ther is no statistical significant difference between any group $(\mathrm{p}=0.7274)$.

Key.

$\mathrm{b}=$ blue, $\mathrm{r}=\mathrm{red}, \mathrm{d}=$ no loght, $\mathrm{Lda}=$ low dose aspirin $(0.325 \mathrm{md} / \mathrm{dL})$; hda $=$ high dose aspirin $(0.925 \mathrm{mg} / \mathrm{dL})$;

hip=hippuric acid $(0.325 \mathrm{mg} / \mathrm{dL})$ 
meric signaling glycoprotein (MolWt VEGF monomer $21 \mathrm{KDa}$ and dimer $42 \mathrm{kDa}$ ) with a structural homology to platelet derived growth factor (PDGF) that stimulates the formation of new blood vessels.

Under the influence of blue light, hippuric acid expressed higher levels of VEGF measuring $114.7 \pm 3.3 \mathrm{pg} / \mathrm{ml}$ compared to the dark sham control value of $105.79 \pm 8.4 \mathrm{pg} / \mathrm{ml}(\mathrm{P}=0.0012)$, while the low and high dose aspirin did not show any significant change. These results clearly show that hippuric acid can induce increased VEGF expression under the influence of blue light when compared to darkness alone; Although not statistically significant, there was a trend demonstrating that cells exposed to hippuric acid and blue light had increased levels of VEGF expression when compared to cells exposed to blue light alone, low dose aspirin, and high dose aspirin. The same trend is observed in cells exposed to darkness, but not red light. Although hippuric acid $\left(\mathrm{C}_{6} \mathrm{H}_{5} \mathrm{CON}-\right.$ $\mathrm{HCH}_{2} \mathrm{COOH}$ ) with a molecular weight of 179.18 Daltons is not a direct metabolite of aspirin, the metabolites of hippuric acid and benzoic acid are common to the metabolites of aspirin (see Figure 2). It is possible that biodegradation of either aspirin or hippuric acid may generate the same metabolite that can trigger the expression of VEGF, particularly polyhydroxylated benzoic acids such as 2,3-dihydroxybenzoic acid. Furthermore, following toluene metabolism, hippuric acid, the glycine conjugate of benzoic acid could be generated in the body and later excreted in the urine. Measurement of hippuric acid in urine could be used as a screening test for individuals exposed to toluene in the absence of xylene and styrene $[20,21]$. People may be exposed to styrene and xylene in the workplace. Furthermore, the ingestion of sodium benzoate, a very common food preservative, salicylic acid or aspirin by anyone can generate the production of hippuric acid. A careful history taken from patients with age-related macular degeneration may identify individuals exposed to aspirin, benzoic acid, and other compounds such as styrene and xylene in the workplace. Interestingly, under the influence of oxidative stress provided by blue light in this case, the levels of induced VEGF expression may increase, causing neovascularization and resulting in macular degeneration. It is interesting to note how blue light causes oxidative stress and contributes to the development of macular degeneration. Recent reports show that light emitting diode blue light can damage photoreceptor cells in culture [14]. Given that human eyes are increasingly exposed to light emitted from the light emitting diode (LED) of video display terminals (VDT) containing blue light, it is quite obvious that the pathogenesis of AMD progresses with retinal photic injury caused by excessive light exposure resulting in oxidative stress [22, 23]. Perhaps, increased reactive oxygen species is a cellular mechanism that provides a possible explanation to the clinically observed phenomenon of asthenopia, which is commonly referred to as eye fatigue, after long periods of exposure to LED blue light or VDT blue light. Kuse Y, et al recently demonstrated that in vitro exposure to blue LED light damaged the $661 \mathrm{~W}$ cells and that this damage was associated with rapid ROS increase, NF-kB activation, P38 activation, ERK 1/2 inactivation, S-opsin aggregation and activated caspase-3/7, and autophagy [14] (see Figure 1). In our study, although we did not measure apoptotic or autophagic activity, hippuric acid in the presence of blue light and darkness induced increased expression of VEGF, suggestive of its role in wet AMD.

Our study is the first study which provides evidence of increased expression of VEGF due to the effects of hippuric acid and its metabolites. Under the influence of red light, the low dose, high dose aspirin and hippuric acid groups expressed lower levels of VEGF compared to the values under blue light. Red light is known to reduce oxidative stress. Recently, red was reported to reduce oxidative stress and preserve function in central nervous system tissue vulnerable to secondary degeneration following partial transection of the optic nerve [15]. In our study, hippuric acid in the presence of red light did not induce the expression of VEGF, suggesting that decreased oxidative stress minimizes or has a palliative effect on the development of AMD. Furthermore, it is shown that hippuric acid by itself causes increased oxidative stress and that blue light is unable to rescue this oxidative effect; however, red light is able to rescue this oxidative effect, as evidenced by decreased VEGF secretion. Although we have tested aspirin and hippuric acid, future experiments could assess the effects of other metabolites such as 2-3-dihydroxybenzoic acid and other hydroxybenoic acids that are common metabolites to both aspirin and hippuric acid. Clinically, the next rational approach is to show increased VEGF expression in patients taking aspirin who have developed AMD.

Even though we did not perform assays to quantify activation of apoptotic cellular machinery, we were able to show that there is a non-statistically significant difference in the number of cells surviving exposure to low dose aspirin, high dose aspirin, and hippuric acid under blue light, red light, or in darkness. These data suggest that exposure to blue light, red light, darkness, low dose aspirin, high dose aspirin, and hippuric acid did not have an effect on the survival of RPE cells after 16 hours. Perhaps, there was no difference in gross survival of RPE cells due to the short time frame of the experiment. Alternatively, similar rates of cell survival by the end of the assay could also have been due to the total number of reactive oxygen species not being enough to saturate anti-oxidative biochemical pathways, therefore permitting cell survival. Future studies could utilize more sensitive assays to quantify the degree of apoptosis and generation of reactive oxygen species for an extended period of time under similar experimental conditions.

\section{Conclusion}

Hippuric acid causes a statistically significant increased induction of VEGF secretion in RPE cells exposed to blue light or darkness, suggestive of a cause for neovascularization in age-related macular degeneration due to common metabolites of aspirin and hippuric acid. Red light, known to abrogate oxidative stress, did not have an increased induction of VEGF secretion from basal levels when exposed to hippuric acid. Caution should be exercised in prescribing aspirin to vulnerable populations under increased oxidative stress. The results of this study provide a clear rationale for the use of anti-VEGF therapy, such as bevacizumab and ranibizumab for slowing the progression of neovascular AMD, especially in patients who have been exposed to aspirin for long periods of time. Further large-scale clinical studies are warranted in order to validate these results.

\section{Acknowledgement}

The authors wish to acknowledge research funding received from The Illinois Society for the Prevention of Blindness (ISPB) and The Richard A. Perritt Charitable Foundation. 


\section{References}

[1]. Hyman L (1987) Epidemiology of eye disease in the elderly. Eye 1(Pt 2): 330-341.

[2]. Access Economics (2010) AMD Alliance International. The Global Economic Cost of Visual Impairment.

[3]. Ding X, Patel M, Chan CC (2009) Molecular pathology of age-related macular degeneration. Prog Retin Eye Res 28(1): 1-18.

[4]. Yang Z, Stratton C, Francis PJ, Kleinman ME, Tan PL, et al. (2008) Toll-like receptor 3 and geographic atrophy in age-related macular degeneration. $\mathrm{N}$ Engl J Med 359(14): 1456-1463.

[5]. Weih LM, VanNewkirk MR, McCarty CA, Taylor HR (2000) Age-specific causes of bilateral visual impairment. Arch Ophthalmol 118(2): 264-269.

[6]. Klaver CC, Wolfs RC, Vingerling JR, Hofman A, de Jong PT (1998) Agespecific prevalence and causes of blindness and visual impairment in an older population: the Rotterdam Study. Arch Ophthalmol 116(5): 653-658.

[7]. Seddon JM, George S, Rosner B (2006) Cigarette smoking, fish consumption, omega-3 fatty acid intake, and associations with age-related macular degeneration: the US Twin Study of Age-Related Macular Degeneration. Arch Ophthalmol 124(7): 995-1001.

[8]. Klein BE, Klein R, Lee KE, Moore EL, Danforth L (2001) Risk of incident age-related eye diseases in people with an affected sibling: The Beaver Dam Eye Study. Am J Epidemiol 154(3): 207-211.

[9]. Eells JT, Henry MM, Summerfelt P, Wong-Riley MT, Buchmann EV, et al. (2003) Therapeutic photobiomodulation for methanol-induced retinal toxicity. Proc Natl Acad Sci 100(6): 3439-3444.

[10]. Gustavsson C, Agardh E (2004) Macular infarction after transpupillary thermotherapy for subfoveal choroidal neovascularization in age-related macular degeneration - a possible association with systemic inflammatory disease? Acta Ophthalmol Scand 82(1): 100-102.

[11]. Venza I, Visalli M, Cucinotta M, Teti D, Venza M (2012) Association between oxidative stress and macromolecular damage in elderly patients with age-related macular degeneration. Aging Clin Exp Res 24(1): 21-27.

[12]. Weissman D, Binder CJ (2012) The innate immune response to products of phospholipid peroxidation. Biochim Biophys Acta 1818(10): 2465-2475.

[13]. Fletcher AE, Bentham GC, Agnew M, Young IS, Augood C, et al. (2008) Sunlight Exposure, Antioxidants and Age-Related Macular Degeneration. Arch Ophthalmol 126(10): 1396-1403.

[14]. Kuse Y, Ogawa K, Tsuruma K, Shimazawa M, Hara H (2014) Damage of photoreceptor derived cells in culture induced by light emitting diode-derived blue light. Sci Rep 4: 5223.

[15]. Fitzgerald M, Bartlett CA, Payne SC, Hart NS, Rodger J, et al. (2010) Near Infrared Light Reduces Oxidative Stress and Preserves function in CNS Tissue Vulnerable to Secondary Degeneration following Partial Transection of the Optic Nerve. J Neurotrauma 27(11): 2107-2119.

[16]. de Jong PT, Chakravarthy U, Rahu M, Seland J, Soubrane G, et al. (2012) Associations between aspirin use and aging macula disorders: the European Eye Study. Ophthalmology 119(1): 112-118.

[17]. Liew G, Mitchell P, Wong TY, Rochtchina E, Wang JJ, et al. (2013) The Association of Aspirin use with age-related macular degeneration. JAMA Intern Med 173(4): 258-264.

[18]. Klein BE, Howard KP, Gangnon RE, Dreyer JO, Lee KE, et al. (2012) Longterm use of aspirin and age-related macular degeneration. JAMA 308(23): 2469-2478.

[19]. Wu Y, Zhu W, Li YH, Yu J (2013) Aspirin and Age Related Macular Degeneration; the Possible Relationship. Med Hypothesis Discov Innov Ophthalmol 2(3): 59-68.

[20]. NIOSH (1980) Method P\&CAM 327. NIOSH manual of analytical methods (NMAM). (2nd edtn), Department of Health and Human Services, USA. 6: 80-125.

[21]. NIOSH (1994) Method 8300: Hippuric Acid in Urine. NIOSH manual of analytical methods (NMAM). (4th edtn), Department of Health and Human Services, USA. 2(1-3).

[22]. Shahinfar S, Edward DP, Tso MO (1991) A pathologic study of photoreceptor cell death in retinal photic injury. Curr Eye Res 10(1): 47-59.

[23]. Beatty S, Koh H, Phil M, Henson D, Boulton M (2000) The role of oxidative stress in the pathogenesis of age-related macular degeneration. Surv Ophthalmol 45(2): 115-134 